\title{
El síntoma de la estatalidad: controles de precios y las capacidades estatales. El caso de Precios Cuidados en Argentina
}

The Symptom of Statehood: Price Control Policies and State Capabilities. The Case of Precios Cuidados in Argentina

Juan Pablo Quiroga Licenciado en (Universe la Comunicación Sochl Magister en Políticas Públics y Gerenciamiento del Desarrollo (Georgetown University / UNSAM) Candidato al Doctorado en Ciencias Sociales por la Facultad Latinoamericana de Ciencias Sociales (FLACSO)

Fecha de recepción

27.5.17

Fecha de aceptación 24.8.17

\section{Resumen}

Las denominadas políticas de control de precios han sido tradicionalmente entendidas en término de las preocupaciones por el rol y el tamaño de la burocracia; su eficacia de corto plazo y la ineficacia de mediano-largo y la constitución de mercados negros, entre otros. Sin embargo, poco se ha estudiado su contribución al desarrollo de las capacidades estatales. El presente trabajo buscará analizar el primer año de funcionamiento del programa Precios Cuidados en Argentina, a los efectos de entender en qué medida nuevas formas de articulación público-privada contribuyen a ampliar las capacidades del Estado y sus agencias. Una política pública que nos brindará la posibilidad de analizar los densos vínculos entre Estado, mercado y sociedad.

Palabras clave: control de precios - capacidad estatal - autonomía estatal - estatalidad - Argentina.

Abstract

The so-called price control policies have traditionally been understood in terms of concerns about the role and size of bureaucracy; Its short-term efficacy and its the 
medium-long term inefficiency; and the constitution of black markets; among others. However, little has been researched about its contribution to the development of state capacities. This paper will analyze the first year of Precios Cuidados program in Argentina, in order to understand to what the extent new forms of public-private articulation contribute to expansion of state capabilities. In a word, a public policy that will give us the unique possibility of analyzing the dense links between State, market and society.

Key Words: price control - state capacity - state autonomy - statehood - Argentina

\section{Introducción}

Las denominadas políticas de control de precios han sido tradicionalmente entendidas en término de las preocupaciones por (a) el rol y el tamaño de la burocracia; (b) la eficacia (potencial) de corto plazo y la ineficacia de mediano-largo de los controles; (c) la evasión y la constitución de mercados negros; (d) la eventual violencia emergente tras el desmantelamiento de los controles o subsidios a los bienes de consumo; (e) su potencial impacto en la coalición de gobierno; (f) los niveles de entrega o incluso eventuales quiebres en las cadenas de abastecimiento; ( $\mathrm{g}$ ) los incentivos/distorsiones en la distribución de recursos, como fruto de la alteración del sistema de precios; (h) la búsqueda de alternativas focalizadas con miras a optimizar el uso de recursos a partir de limitar el denominado free-riding; e incluso $(\mathrm{k})$ su devenir histórico como respuesta al alza de precios (Adams 2000; Agendor y Asilis, 1997; Alderman 2002; Bienen y Gersovitz 1986; Colander 1984; Dunn y Pressman 2005; FIEL,1990; Friedman \& Friedman 1990; Galbraith 1941, 1946, 1951, 2001; Rockoff 1984; Schuettinger y Butler, 1978; Taussig, 1919). Sin embargo, poco se ha estudiado su contribución al desarrollo de las capacidades estatales.

El presente trabajo busca aproximarse a este problema por la vía del análisis del primer año de funcionamiento del programa de Precios Cuidados, vigente en Argentina desde el 06 de enero de 2014. Inspira esta investigación la idea que dicho estudio podría ayudar a entender en qué medida formas relativamente novedosas de articulación entre el sector público, el privado y las organizaciones sociales pueden contribuir, en determinadas circunstancias, a ampliar las capacidades estatales, a partir -fundamentalmente- de cuatro mecanismos muy precisos: la articulación con redes informales de monitoreo, control y sanción; la intervención de forma directa del piso de venta de las principales cadenas de comercialización; el incremento en el flujo de intercambio de información del sector privado a las agencias de gobierno involucradas; $y$ la transferencia indirecta de recursos del sector privado a la operación del programa, en un proceso más amplio de disciplinamiento del empresariado.

Por otra parte, sostendremos que el sistema de precios, así como su regulación/desregulación, constituye un objeto privilegiado para el análisis de la relación, no siempre explícita pero siempre presente, entre el Estado y su otredad: las estructuras de mercado.

En este sentido, partimos de entender al Estado no sólo como un agregado heterogéneo de agencias de gobierno, con distribuciones diferenciales entre las mismas de recurso y capacidades para la acción, sino también como un actor clave en lo económico tanto en lo relativo al proceso de acumulación de capital, como de transformación del perfil productivo de un país y la redistribución del ingreso (Evans y Rueschemeyer 1985). Después de todo, “(...) la creación de la riqueza no es ya solamente una función de la naturaleza o del mercado; sino que implica también un estructura estatal efectiva" (Evans 1995:6), en sociedades donde -cada vez más- el Estado debe garantizar, a su vez, niveles aceptables de bienestar. O bien , por lo menos, establecer incentivos a partir de la definición de ciertas reglas de juego, con el objetivo de reducir la incertidumbre, dotar de estructura y patrón a las interacciones diarias, minimizar el conjunto de opciones disponibles y reducir/incrementar los costos de transacción y producción (North 1990).

En una palabra, los mercados no son, entonces, la emergente de un curso "natural" de las cosas, sino un fenómeno político en donde el Estado cumple una función decisiva. En este punto, la intervención estatal no constituye -simplemente- un esfuerzo por "proteger" a la sociedad del mercado, sino una fuerza clave del desarrollo de este último. El Estado oscila, en este sentido, en una tensión que “(...) define [su] naturaleza contradictoria” (Alonso 2000:25), en la búsqueda por producir un equilibrio dinámico entre la necesidades de acumulación y su legitimación como fruto de la intervención socio-política. De hecho, la "función de acumulación" suele ser señalada en la literatura como la condición misma de funcionamiento del aparato estatal, en vistas a que de ella dependen las capacidades extractivas y la posibilidad de expansión de las intervenciones estatales.

En otros términos, "una maquinaria burocrática efectiva es clave en términos de la capacidad de intervención del Estado. Sin embargo, para que el Estado capitalista pueda involucrarse en la transformación económica, el funcionamiento de la mencionada maquinaria debe articularse con el funcionamiento del mercado. En un punto, el análisis sobre las estructuras estatales deben unirse con los análisis de las estructuras de mercado" (Evans y Rueschemeyer 1985:51).

Se hace evidente, entonces, la trama de relaciones entre Estado, mercado y sociedad. 
Una trama que el presente trabajo nos brindará la posibilidad de analizar, al poner el acento en la regulación del mercado de bienes de consumo básico.

Por último, si bien el trabajo se centrará en el caso argentino, algunas referencias y comparaciones con el programa de "Precios Justos", desarrollado en Venezuela en la misma época, serán de suma utilidad a los efectos de entender la especificidad y el estatuto particular de la articulación entre Estado y sociedad prevista en la política pública bajo análisis.

\section{Las políticas de control de precio}

En Argentina las políticas de control de precio nacieron formalmente en 1939 como medida de excepción en el marco mismo de la Segunda Guerra Mundial, ante la amenaza de alteración de los flujos de comercio internacional.

En este contexto, el Congreso sancionó la Ley 12.591, la cual sería prorrogada de forma recurrente en 1952 (Ley 14.120), 1955 (Ley 14.440 y Ley 12.830), para -a partir de la Ley Nacional de Abastecimiento de 1964- pasar a ser fundada no ya en un régimen de excepción sino en una función más del Estado. Desde entonces, se sucederán varias leyes destinadas a reglar estas facultades: en 1966 se sancionaría la Ley 17.017 y tras su vencimiento la Ley de Ordenamiento Económico 18.691/70, hasta que en 1974 se sancionaría la Ley 20.680 o Ley de Abastecimiento en vigencia hasta su modificación en 2014.

Asimismo, en el período comprendido entre 1967 y 1989, por ejemplo, los controles de precio serían la norma, mientras que los lapsos de "libertad de precios" quedarían limitados a 30 meses, sobre un total de 274 (Fiel 1990). Un dato que lleva a los autores del mismo estudio a plantear que -justamente- fue su institucionalización como régimen normal y no de excepción, lo que erosionó la credibilidad sobre los mismos.

Incluso, se trataría de una problemática -al menos en lo referente al caso argentinohistóricamente sensible en lo relativo a las alianzas políticas. De hecho, las políticas de control de precios de productos de primera necesidad constituyeron una arena clave en materia de regulación, en vistas a los efectos y consecuencias inherentes a la relación exportable-alimento-bien-salario (O'Donnell 1977). Sobre todo, a la luz de dos dilemas: la emergencia en Argentina de un sector popular con recursos económicos y organizativos significativamente mayores al resto de la región; y el hecho que los principales productos de exportación de la economía sean aquellos que constituyen el principal bien-salario de los sectores populares, en donde un cambio en sus precios relativos tiende a ser inmediatamente percibido. Una relación entre exportable-alimento-biensalario que habiendo sido teorizada por O'Donnell como la base objetiva para repetidas alianzas entre buena parte de las fracciones débiles de la burguesía urbana y el sector popular, hoy es discutida por otros autores, a la luz del boom de la soja (Richardson 2009).
Ahora bien, de la revisión bibliográfica y el devenir social del debate sobre las políticas de control de precios, se evidencia una serie de invariantes. En primer lugar, se hace manifiesto un conjunto de referencias semánticas y problemáticas propias y recurrentes, entre ellas: la preocupación por el rol y el tamaño de la burocracia; la eficacia de corto plazo y la ineficacia de mediano-largo de los controles; la evasión y la constitución de mercados negros; la violencia emergente tras el desmantelamiento de los controles o subsidios a los bienes de consumo; su potencial impacto en la coalición de gobierno; las preocupaciones por los niveles de entrega en las cadenas de abastecimiento; el impacto económico de los controles en la economía local; y los incentivos/distorsiones en la distribución de recursos, como fruto de la alteración del sistema de precios.

A su vez, una segunda serie de invariantes, estaría dada por la estabilidad (relativa) de las formas que asumieron los controles en el tiempo. De hecho, de la literatura se desprende una tipología de cinco hechos estilizados en materia de controles de precios directos. En primer lugar, los "precios vigilados" en donde la atención se centra en las cadenas de comercialización, las cuales deben avisar a la Secretaría de Comercio sobre eventuales cambios en los precios. En segundo término, el denominado "acuerdo de precio", por el cual empresas comercializadoras y proveedores pactan los valores de un set de productos. Una tercera opción, en cambio, es denominada, con frecuencia, "precios controlados" y consiste la necesidad por parte de productores y comercializadores de obtener aprobación de la Secretaría de Comercio para realizar cambios de precio. En cuarto lugar, existirían los "precios máximos" por los cuales la autoridad competente fija precios máximos para determinados productos; mientras que, por último, en quinto lugar residiría el "congelamiento generalizado" por un tiempo determinado, en los cuales el gobierno restringe de forma absoluta los cambios en materia de precios.

Por último, existe una tercera propiedad invariante en el análisis, de suma importancia a los efectos de nuestra investigación, la cual sólo se hace evidente por su omisión en el debate: la discusión sobre las capacidades (presuntas) del Estado. En este punto, es interesante constatar que toda la discusión sobre las políticas de control de precios da por hecho, como constante, la existencia de capacidades para su realización. Las diferencias, en cambio, sólo radicarán en el acento valorativo (+/-) en que se enmarque mencionada intervención, pero nunca son puestas en duda en su existencia. Es decir, o bien el Estado tiene capacidades para controlar los precios, o bien para distorsionar el sistema de mercado, pero bajo ningún punto no tiene capacidades: en el peor de los escenarios, resultan ineficientes, pero nunca no-son.

En este marco, se hace evidente un déficit en la teoría, en vistas a que poco se ha estudiado la contribución de las políticas de control de precio al desarrollo de las capacidades estatales. Se trata, en suma, de un caso particular, de un fenómeno general que ha sido denominado "policy feedback" (Pierson,1993) y que consiste en considerar a las 
políticas públicas no sólo como outputs, sino también inputs del proceso político. Incluso, algunos estudios se han realizado en esta línea, como el desarrollado por Ryan Saylor, quien plantea que el boom de exportación de commodities en Chile a finales del 1800 posibilitó la expansión de las capacidades del Estado, a partir de la provisión de bienes públicos y el desarrollo de instituciones que protegieran los intereses creados por parte de los grupos económicos dominantes (Saylor, 2012).

\section{La "forma" Precios Cuidados}

En enero de 2014, y en el marco de un crecimiento de las expectativas inflacionarias, así como de una devaluación del orden del 40\%, nace el Programa de Precios Cuidados, como parte de un acuerdo entre la Secretaría de Comercio (dependiente del Ministerio de Economía de la Nación), los supermercados y sus principales proveedores para la administración de los precios de un set (inicial) de 194 productos durante todo 2014.

El mismo tendría por objeto tres objetivos declarados: (a) asegurar condiciones de competitividad en la economía, (b) cuidar el bolsillo de los argentinos, y (c) que cada consumidor pudiera ejercer su derecho de elegir informado. Es decir, alineado con la definición de la cuestión, se trataría de una respuesta pública a un (presunto) problema de ambición privada y márgenes de ganancia, en distintos puntos de la estructura de costos, tanto de los sectores productivos como de distribución. El diseño del programa buscaría establecer y dar visibilidad a una canasta de productos con "precios de referencia" que contemplen "márgenes razonables" de rentabilidad.

En su etapa inicial, el programa buscó administrar el precio de 194 productos, producidos por 75 empresas proveedoras y distribuidos por 10 cadenas de supermercados de todo el país. Sin embargo, iría incorporando nuevos ítems, como fruto de las revisiones trimestrales, hasta llegar al día de hoy -al momento de escritura del presente- a administrar una canasta total de 455 productos, con aumentos en los períodos mencionados del orden del $3 \%$ en promedio.

El programa sería rápidamente aceptado, registrando un alto nivel de conocimiento y de valoración. De hecho, según una encuesta nacional de IPSOS-Mora y Araujo, ${ }^{1}$ el 96\% de los consultados en el mes de noviembre de 2014 manifestó conocer el programa y su alcance. Incluso, el 75\% del total de los mismos, a nivel nacional, declaró -en el mismo período- haber buscado al menos un producto del programa, siendo el $81 \%$ de ellos responsables principales de las compras del hogar.

Asimismo, determinadas categorías de productos previstos por el programa, se perfilarían como la principal opción en el orden de preferencias de los clientes: en referencias de almacén, por ejemplo, el 74\% de los consultados declararía buscar productos de Pre- cios Cuidados como primera opción, mientras que ese número llegaría al 69\% en lácteos y $66 \%$ en productos de limpieza.

Por otro lado, la demanda durante el primer año se multiplicaría -en promedio- siete veces, a la vez que en el mismo período, según fuentes del sector, los productos que forman parte del programa habrían pasado de representar el 5\%, al 20\% de la facturación de las grandes cadenas minoristas locales.

El andamiaje jurídico del programa -por otra parte- supondría un elemento distintivo. En este sentido, el acuerdo daba cuenta de dos series de convenios en paralelo. ${ }^{2}$ En primer lugar, aquel firmado entre la Secretaría de Comercio Interior (SCI) y las cadenas de supermercados, donde las mismas aceptan de forma voluntaria comercializar y poner a la venta los productos definidos, al precio acordado; mientras el segundo, en cambio, sería firmado entre la SCI y cada proveedor de los productos previstos en la canasta, donde éstos últimos se comprometían a garantizar los niveles de entrega y el precio establecido.

La novedad de la arquitectura legal prevista por el programa radicaría en la economía jurídica propuesta: estamos ante una serie de diez cláusulas breves, de suscripción voluntaria, sustentadas sobre un corpus jurídico de referencia compuesto por normas preexistentes: el artículo 42 de la Constitución Nacional, el cual establece los derechos de los consumidores y usuario de servicios; la Ley 22.802 de Lealtad Comercial; la Ley 24.240 de Defensa del Consumidor; la Ley 25.156 de Defensa de la Competencia; y el Decreto 2136/2013 en donde se fijan los objetivos de la SCI. Es decir, por fuera del acuerdo voluntario de diez artículos, no se necesitaron nuevos elementos jurídicos, con los costos (en términos de alianzas, negociación, debate con actores clave, entre otros) que esto hubiese implicado.

Ahora bien, en lo que a nosotros respecta, existen cuatro nudos jurídicos previstos por el acuerdo de especial importancia a los efectos del presente trabajo. En primer lugar, el mismo regiría únicamente para aquellos productos dados de alta a la fecha de firma del acuerdo. Es decir, las cadenas no tendrían la obligación de sumar productos que aún previstos en la canasta del acuerdo no comercializaran al momento de la firma del mismo. Posibilitando, de esta forma, que pudiesen concentrar esfuerzos en disponer en cantidad de lo que ya tenían y no ampliar su base de surtido.

Asimismo, las partes se comprometían a revisiones trimestrales de precios, con la excepción de los productos de verdulería y carnes, los cuales serían sujetos a revisión mensual a pedido del supermercado, sobre todo por la estacionalidad de los mismos.

En tercer término, un punto importante radicaría en la posibilidad de informar a la SCI de forma inmediata, a través del denominado "sistema de alerta temprana", cuando se 
verifiquen irregularidades en los niveles de entrega por parte de los proveedores. En el caso en que se reiterase el faltante, el producto podía ser reemplazo de común acuerdo por otro de similares características.

Por último, las empresas comercializadoras asumirían la responsabilidad por la señalización diferencial de los productos. Un sistema que mantendría características similares para todos, definidos por la Secretaría de Medios de la Nación, pero que sería objeto de multas en caso de no encontrarse conforme a lo estipulado.

Este acuerdo inicial tendría una vigencia de un año, pero sería refrendado de forma trimestral, a través de sucesivas adendas, con el objeto de actualizar los precios de los productos incluidos, a la vez que extender la misma a una mayor cantidad de referencias. No obstante, la primera de las adendas verificaría -además- dos novedades: en primer lugar, se segmentaría la canasta por formato. Un hecho que permitiría a las grandes cadenas no disponer del total de productos en todos los tipos de sucursales, sino distinguir entre hipermercados (con más de 5.000 metros cuadrados y 50.000 referencias a la venta) y formatos de cercanía (de 200 metros cuadrados), donde sólo se comercializarían cerca de 5.000 productos.

Por otro lado, se fijaría una línea de cumplimiento del orden del 80\%, lo que posibilitaría que las multas, actas u observaciones se efectúen sobre la totalidad de la implementación del programa en un local y no en función de la presencia/ausencia o mala señalización de un producto puntual.

Comienzan, poco a poco, a hacerse evidentes dos hechos sustanciales. En primer lugar, la ejecución de Precios Cuidados se sostendría sobre una economía jurídicoadministrativa sorprendente. Es decir, se estructuraría sobre la base de un convenio firmado con las principales cadenas comercializadoras y productores, y una serie de sucesivas adendas las cuales extenderían tanto el plazo de vigencia, como el alcance del programa. En segundo, las agencias de gobierno, fundamentalmente la SCI, contemplaría las demandas del empresariado minorista local, sobre todo en lo relativo al abastecimiento, la necesidad de revisión de precios, un diferencial de aumento por región del país por los costos logísticos implicados en la comercialización y la segmentación del surtido. Es decir, que no fuese necesario disponer de todo, en todo momento y en todos los formatos.
Figura 1 Diferencias entre las modalidades de Precios Cuidados (Argentina) y Precios Justos (Venezuela)

\begin{tabular}{|l|c|c|}
\hline & Modalidad Venezolana & Modalidad Argentina \\
\hline Instrumento legal & Ley, modificaciones y resoluciones & Convenio sectorial \\
\hline Actores implicados & Toda el funcionamiento de la economía & $\begin{array}{c}\text { Gobierno y sectores productivos y de } \\
\text { comercialización }\end{array}$ \\
\hline Productos & Todos los bienes y servicios & 194 productos \\
\hline Régimen de articulación & $\begin{array}{c}\text { Compulsiva (no hay producto que no } \\
\text { esté regulado) }\end{array}$ & $\begin{array}{c}\text { Optativa (el consumidor puede optar por } \\
\text { productos por fuera de la canasta) }\end{array}$ \\
\hline Objeto & Fijar techos & $\begin{array}{c}\text { Administrar precios de una canasta limitada } \\
\text { y ofrecer precios de referencia }\end{array}$ \\
\hline
\end{tabular}

Fuente: Elaboración propia

En contraposición a estos dos atributos (economía jurídica y sensibilidad ante ciertas demandas de los operadores locales) el gobierno de Venezuela, por ejemplo, presentaría al mismo tiempo (en enero de 2014) el programa de Precios Justos, el cual implicaría una batería legal más compleja y densa a los efecto de asegurar el rol del gobierno de fijar precios máximos de todos los bienes disponibles en el mercado de consumo, a partir de establecer un techo del 30\% a los márgenes de ganancia; y fiscalizar su implementación, al punto de poder expropiar mercadería y venderla de forma directa al público. De hecho, en su primer año de implementación se sancionó una ley ("Ley Orgánica de Precios Justos”); una reforma a la misma, para incorporar más artículos; y diez resoluciones, las cuales además de operacionalizar la ley marco, dictaban adecuaciones sobre faltantes, como leche, jabón, arroz, maíz, entre otros.

Adelantándonos en nuestra argumentación, el cuadro anterior pone de relieve que la diferencia sustancial entre ambas políticas, radicaría en que mientras Precios Justos se enfrentó al empresariados a partir de la movilización de todo el aparato del Estado, en el marco de lo que será entendido y conceptualizado como una "Guerra Económica"; la "forma" particular de Precios Cuidados, en cambio, pondría en acto instancias de "disciplinamiento" del empresariado, en los términos de Amsden (1992): un componente central de la reciprocidad en la relación entre Estado y empresarios, donde se mantienen canales de diálogo con el sector privado y se institucionalizan (de forma incipiente) instancias de comunicación, sin necesidad de recurrir a mecanismos de coerción directa (garantizadas, fundamentalmente, por la necesidad de negociar de forma periódica los términos y alcances del programa, así como reportar de forma semanal los niveles de abastecimiento).

Ahora bien, en relación a los resultados del programa, en tanto que “(...) cambios observables en las condiciones de vida de la población" (Bertranou, 2013:18), según datos oficiales la participación de los productos de Precios Cuidados en la facturación de los supermercados ascendió del 5\% al 20\% (“Se lanzó la cuarta etapa...”, 2014). Un dato que las fuentes consultadas en el sector privado ratifican y ubican -según la cadena en cuestión- entre el 14\% y el 18\%. 
La demanda de los productos integran la canasta del programa aumentó $700 \%$ promedio en los dos primeros meses de vigencia, , los mismos evidenciaron, en dos meses, un aumento del $700 \%$ promedio en su demanda, según los empresarios entrevistados. Conforme lo mencionado por los mismos, tomando el mes de abril de 2014 y comparándolo con el mismo mes de 2013 (en donde no existía le programa), los productos de Precios Cuidados verificaron un incremento en la facturación del 159\%. De hecho, según una de las cadenas, comparando la demanda en unidades de la canasta en febrero de 2015 contra febrero de 2013 (cuando no existía el programa), la venta de los productos en cuestión habría subido un $21 \%$. Si la comparación, en cambio, se hiciese entre febrero de 2015 y febrero de 2014 -es decir, entre el trigésimo mes de funcionamiento del programa y el segundo), el aumento en ventas de unidades sería del $20 \%$ sobre los items comparables. ${ }^{3}$

Por otra parte, los niveles de abastecimiento habrían oscilado en torno al $65 \%$ en promedio, dependiendo de las categorías en cuestión. Es decir que, de cada orden de 10 productos, los productores entregaban en las sucursales 6.5 en promedio, siendo las categorías más sensibles aquellas vinculadas con aceite, yerba, azúcar y harina, por mencionar algunas.

Por otro lado, según el Estudio de Faltantes de Mercadería de Góndolas publicado por la organización GS1, la cual reúne a las principales cadenas y empresas proveedoras del sistema de códigos de barras, la provisión de mercadería en 2014 habría registrado un pico histórico de desabastecimiento de 19,47\%, cuando el promedio de los años 2011 , 2012 y 2013 rondaba un 13,44\%. El mismo valor verificado a fines del 2015. En cuanto a las causas de los mismos, el 40,70\% de los casos obedecerían a que la mercadería no fue repuesta en góndola; en el 20,02\% a que el proveedor no entregó el producto; en el 19,20\% a que el local no realizó ajustes del inventario; en el 9,07\% a que el centro de distribución no había modulado los pedidos; y en el 3,48\% a que la sucursal no trabaja el producto. A su vez, el mismo informe señala que el $43 \%$ del total de los faltantes dentro de las categorías de Precios Cuidados pertenece al rubro de alimentos, mientras el 20\% a Cuidado del Hogar, 19\% a Bebidas y $18 \%$ a Cuidado Personal.

Frente a estos datos, y en otro nivel de análisis del orden del impacto agregado, puede decirse que estamos ante un programa que configura, en la tipología de Lowi (Birkland 2005), una política pública a la vez regulatoria y redistributiva. En primer lugar, porque buscaba proteger a la sociedad de las externalidades negativas de la concentración tanto de la producción como de la distribución de bienes de consumo masivo y su impacto en la estructura de costos. En segundo término, en cambio, se trata de una política redistributiva en la medida en que supone, como base de su funcionamiento, una transferencia de recursos de sectores concentrados al conjunto de potenciales clientes. Los cuales, incluso, por definición cubren al conjunto de la sociedad al incluir en la canasta primeras marcas (como Coca-Cola, Unilever, P\&G, y Nestlé) y una dispersión de productos que van desde leche hasta preservativos y yogures light de marcas líderes de mercado. Este carácter de universalidad potencial de la política, por los tipos de productos que contempla, es la condición misma de posibilidad del crecimiento en la demanda de los mismos, así como es la contracara de las políticas auto-focalizadas (self-targeted) en donde el elemento subsidiado es un bien "inferior", consumido preferencialmente por los sectores populares (Adams 2000:4)

En términos de un empresario perteneciente a uno de los principales grupos alimenticios de Argentina: "el programa fue un éxito y está claro que es uno de los logros que puede mostrar el equipo económico. Precios Cuidados provocó una variación positiva para la gran mayoría de los productos participantes. Pero como contrapartida estamos enfrentando situaciones muy complejas en materia de rentabilidad, especialmente en algunos rubros, como el aceite. Hoy, el aceite tiene un precio irreal, en el que los exportadores están subsidiando a los consumidores locales” (Sainz, 2015).

Incluso, el empresariado realizó una segunda serie de transferencias al funcionamiento del programa, las cuales se sobreimprimen a la renuncia de márgenes de ganancias: como parte de los compromisos asumidos en el marco del acuerdo, las cadenas debían resignar el espacio destinado a la promoción de productos y promociones en el piso de venta para la disposición de cartelería oficial, así como la distribución de folletos con los productos y los precios acordados. Todos materiales y productos producidos y financiados por las mismas cadenas.

Este es un punto central, dado que la intervención del piso de venta (la transferencia al sector público de un espacio tradicionalmente puesto en valor por parte de las cadenas para con sus proveedores) posibilitó la clara identificación del programa, el cual pasó a operar como una marca más: de fácil identificación, con un logo, señalización, folletos, cartelería aérea, listados al ingreso de tienda entre otros. En una palabra, las cadenas debían no sólo renunciar a los márgenes de ganancia y transferir de forma indirecta recursos al funcionamiento del programa, a la vez que incrementar los costos administrativos y de transacción para la ejecución del mismo, sino también asumir aquellos propios de la señalización en piso de venta, tanto por la producción de materiales, como la renuncia a la comercialización de esos espacios. La Secretaría de Medios de la Nación, por su parte, asumiría como propio el diseño del logo y la responsabilidad por la difusión en medios masivos gráficos, radiales y televisivos.

Es esta ejecución, sobre todo, la que será objeto de los controles por parte de las organizaciones sociales y grupos militantes que, sin estar organizados de forma directa por las agencias estatales (a diferencia del caso venezolano), controlarán semanalmente los productos y su señalización, así como realizarán "escraches" a los presidentes de las prin- 
cipales cadenas, y distribuirán panfletos en contra de las denominadas "corporaciones económicas".Volveremos sobre este punto.

\section{Precios Cuidados y las componentes de la capacidad estatal}

Hecho este rodeo a los efectos de definir y establecer la "forma" particular implicada por el programa, volvamos sobre el nudo de nuestra argumentación: el incremento en las capacidades estatales como consecuencia de los arreglos instituciones previstos. En este sentido sostendremos que un incremento en las denominadas "capacidades estatales" debe ser producto de una mejora en alguna o varias de sus dimensiones observables, o cuatro componentes, a partir de los cuales puede operacionalizarse mencionado concepto, conforme los desarrollos de Bertranou (2013). A saber: (a) la legitimidad del sujeto estatal; (b) los vínculos del Estado con actores no-estatales; (c) las características de los arreglos institucionales y la estructura burocrática; y (d) el capital de acción interinstitucional. Analicemos -a continuación- cada uno de ellos.

\subsection{Legitimidad del sujeto estatal}

En lo relativo a la legitimidad del accionar de la Secretaría de Comercio, en particular en lo referente a las autorizaciones legales que sustentan su acción, se destaca una deflación en términos de instrumentos normativos. Como analizábamos en el apartado anterior, la ejecución del programa se sostiene simplemente sobre un convenio firmado con las principales cadenas comercializadoras y productores, y una serie de sucesivas adendas las cuales extenderían tanto el plazo de vigencia, como el alcance del programa.

En cuanto a la legitimidad político-institucional para desplegar su accionar, al implicar un acuerdo con las cámaras empresariales del sector y los empresarios, el caso de Precios Cuidados encontró desde su origen mismo legitimidad para actuar. No obstante, la mayor fuente de legitimidad a la intervención estatal y el despliegue de capacidades, estaría dada por la opinión pública. Según un estudio de IPSOS-Mora y Araujo de más de 998 casos a nivel nacional, en Argentina más del 50\% de la población encuestada se encontraba al momento de la realización del campo a favor que el gobierno controle los precios de los alimentos básicos y servicios. Incluso, el 43\% declaraba que las empresas de consumo masivo deberían estar bajo directa administración del Estado.

En suma, a nivel de la opinión pública, lo que se hacía evidente era la existencia de cierta valoración positiva en relación a la presencia del Estado en la economía, legitimando cualquier posible accionar en este campo, sobre todo en lo relativo al contro de alimentos básicos. En este punto, el lugar de la opinión pública y la presión que ejerce sobre el sistema político y sus instituciones parece ser sintomático. En un país con niveles de institucionalidad críticos y con escasa participación de las élites (políticas, empresariales, sindicales u de otro orden), como sectores dirigentes, sobre los procesos de formación de opinión, no es de extrañar, como sostiene Mora y Araujo, que "para entender a la Argentina [sea] preciso comprender a la opinión pública antes que a sus dirigentes (Mora y Araujo, 2011:17).

\subsection{El vínculo con actores no estatales y el poder de sanción informal}

En este punto, comienzan a evidenciarse las particularidades del caso argentino en lo relativo a la segunda de las componentes de las capacidades estatales: el vínculo del Estado con actores no estatales. Sin exagerar, puede decirse que Precios Cuidados, sobre todo a diferencia de Precios Justos, supone un reconocimiento del hecho que la autonomía del Estado no es absoluta, sino que por el contrario se encuentra enraizada: mantiene una serie de vínculos concretos que la unen a la sociedad a través de canales institucionalizados para la negociación y renegociación continúa de las políticas y sus metas (Evans 1995).

El programa contempla instancias de negociación permanente, no solamente cada tres meses a los efectos extender su alcance, acordar las subas autorizadas de los productos y -eventualmente- incorporar nuevos, sino también contactos diarios con las áreas de abastecimiento de las principales empresas en vistas a que reporten mediante un sistema de "alertas tempranas", los incumplimientos en las entregas y el total de órdenes de compra por producto. Un sistema de reporte en tiempo real que permite a las cadenas de supermercados denunciar el faltante de producto de forma online a la SCI, con miras a que pueda controlarse a los productores frente a un posible quiebre de stock.

Incluso, el diseño del programa contemplaría, como mencionamos más arriba, cambios en función de las preocupaciones del sector en torno al aumento periódico de precios, los costos logísticos para el traslado de los mismos al interior del país y la estacionalidad mensual de frutas, verduras y carnes, entre otros.

En el caso venezolano, en cambio, la relación Estado-sociedad quedará circunscripta no a mesas de negociación institucionalizadas, sino a la confrontación abierta entre ambas esferas. De hecho, mencionada relación sería recurrentemente figurada a partir de ciertos rasgos propios del campo semántico bélico como estar en "guerra", "batalla", "Brigadas juveniles para la defensa popular de la economía" "Comisión de la Paz del Área Económica", etc. Se trata de unidades semánticas que constituyen una forma recurrente de enfatizar los aspectos bélicos a la relación Estado/Sociedad en detrimentos de otros atributos posibles. El modelo venezolano, en suma, parte de entender al Estado y su autonomía como el único lugar de realización de los intereses de la sociedad.

Por otro lado, Precios Cuidados implicará una relación con actores no-estatales en otro sentido aún más decisivo para el funcionamiento del programa: su articulación con 
organizaciones sociales para el control, monitoreo del cumplimiento del programa y sanción. Un tipo de prácticas articulatorias que nos pone frente a la necesidad de reconocer el rol que cumplen estos vínculos en la construcción de la capacidad del Estado (Amengual 2013:309).

En este sentido, la articulación con diversas agrupaciones tiene dos efectos directos. En primer lugar, posibilita un crecimiento de la capacidad para penetrar en la sociedad civil y poner en acto las decisiones; en nuestro caso: que los productos definidos estén en las góndolas, al precio acordado y con la señalización especial definida. En términos concretos, según datos de una de las principales agrupaciones políticas participantes, en el inicio del programa se habrían distribuido más de 500.000 panfletos con los listados de los precios y productos incluidos, y habrían participado -solo de esa agrupación- 1.700 militantes, 400 en la Ciudad de Buenos Aires y 1.300 en diferentes puntos del territorio bonaerense. En esta línea, en la Ciudad de Buenos Aires se instalaron frente a cadenas minoristas como supermercados y autoservicios 460 mesas; mientras que en la provincia se instalaron unas 360 mesas de difusión en puntos estratégicos de consumo masivo.

Esta articulación posibilita una ampliación del margen de la capacidad de control de los solo 41 "relevadores" oficiales que disponía la SCI, asegurando la capacidad de inspección, la cual -de lo contrario- no hubiese alcanzado para cubrir el total implicado de 2.000 bocas de expendio a nivel nacional.

En segundo lugar, esta articulación con actores no estatales, posibilitaría el establecimiento de un régimen de sanciones "no oficiales" vinculadas con cierto accionar ilegal, como el daño a la propiedad privada a partir de pintadas y "escraches", ${ }^{4}$ todas llevadas a cabo por organizaciones sociales y/o políticas.

En una palabra, este tipo de articulación amplía los recursos estatales de sanción, a partir de cierto régimen de "estatalidad híbrida" (Dewey 2012). Es decir, una forma particular de accionar del Estado que contempla el hecho que pueda actuar de forma ilegal, a partir de agrupaciones sociales y políticas en este caso, para responder a demandas sociales (al cumplimiento efectivo del acuerdo). Desde esta perspectiva, las prácticas ilegales e informales forman parte del stock de recursos usados para proveer seguridad a la población.

De esta forma, el stock de recursos punitorios disponibles al alcance del Estado, fundamentalmente inspecciones, multas y clausuras, se ven complementadas con instancias informales.

Este sistema punitorio informal se sobreimprimió en los pliegues mismo del funcionamiento formal del régimen de monitoreo y sanción, el cual se encontraba regulado por tres normativas diferentes las cuales buscan administrar las prácticas en materia de exhibición de precios, de diferencias entre el precio de góndola y el imputado en la caja, y el faltante de stock.
Según los empresarios consultados, de las tres cuestiones susceptibles de ser multadas, el faltante de producto concentraría cerca del $75 \%$ de las multas, mientras que las diferencias de precios entre la señalización de la góndola y el ticket final, rondaría el 20\%. La diferencia, del orden del 5\%, se explicaría por faltas en la exhibición de precios. De hecho, al día de la fecha, se labraron multas por un monto total de AR $\$ 31$ millones de pesos (Franco, 2014), dos de las cuales han sido ratificadas por la Justicia (“Confirman multas...", 2014).

En promedio, las cadenas consultadas habrían recibido por parte de la SCI 150 actas, de las cuales solo el $10 \%$ tuvo -en el plazo considerado- una sanción efectiva. El resto, o bien fueron archivadas (30\%) o bien tienen pendiente su resolución (60\%). Este desfase entre multa efectiva, pendiente y archivada radica en que -como sostienen los informantes- "como cadena, nosotros no estamos obligados a garantizar, el 100\% de stock del 100\% de los productos de forma permanente. Es más... el 70\% de las veces podemos probar que los productos infraccionados fueron oportunamente informados como Alerta Temprana, sin embargo, la Secretaría no justificó debidamente el faltante. Un punto que no nos salva del costo de reputación cuando se publican estos datos en los medios".

En este punto, puede verse que el impacto del programa sobre las operaciones de las empresas radicaría menos en la efectividad de la sanción, que en el alza en los costos de transacción, como fruto del incremento en los gastos administrativos para la implementación y seguimiento del programa, así como la defensa/apelación de las multas y sanciones; y el costo de reputación para con la opinión pública en general y su clientela en particular.

Por último, al conjunto de sanciones formales e informales y la alianza con actores no estatales, debe sumarse una serie de herramientas desarrolladas para el control directo por parte de los consumidores. En este sentido, de cara a la ciudadanía en general, el programa puso a disposición un sistema telefónico y online de denuncia, así como una aplicación para teléfonos móviles que permite el escaneo del código de barra de un producto y así saber si pertenece al set de productos cuidados y cuál debería ser su precio. Incluso, permitía a los usuarios enviar en el momento un mensaje, con foto, a la Secretaría de Comercio para denunciar si el mismo no se encuentra disponible o bien el precio no es correcto.

\subsection{Los arreglos institucionales: el desvío del grado cero}

Por otro lado, en cuanto al tercer componente, el relativo a las características de los arreglos institucionales y la estructura de la burocracia, la novedad del programa en este punto radicó en el desvío que sus arreglos y expresiones organizacionales supusieron de la estructura burocrática verificada en promedio en las agencias estatales nacionales. Sobre todo, en lo relativo a la expresión mínima formal en la que se cristalizaba una estructura de 41 relevadores a nivel nacional, y el apoyo en organizaciones sociales y políticas, tal y como fuera mencionado en el punto anterior, a los efectos del control, monitoreo y sanción. 
De hecho, la "original" propuesta del programa (el hecho que para hacer efectivo y capilar su aparato de control y monitoreo recurra a cierta hibridez) debe ser leída a la luz de cierta desconfianza de la política sobre el nivel de compromiso de las estructuras burocráticas tradicionales para el cumplimiento de objetivos específicos. En palabras de Oszlak, "cada nuevo régimen intenta alterar no sólo las relaciones de poder dentro de la sociedad civil, en línea con su concepción política y la necesidad de fortalecer sus bases sociales de apoyo, sino también la estructura de poder dentro del mismo aparato estatal. Para hacer viable un proyecto político, se requiere actuar sobre -como también mediante- una estructura burocrática preexistente. Aumentar la congruencia entre el proyecto político y la organización pública, puede llevar a cambios jurisdiccionales, jerárquicos y de competencias, afectando intereses establecidos y modificando arreglos de poder y patrones culturales fuertemente enraizados en la burocracia estatal" (Oszlak 2006:14). Es decir, solo la militancia de base ofrecía las condiciones necesarias en términos de verticalidad, conciencia de misión y fines, así como capilaridad para controlar el programa.

No obstante, poder describir este grado cero de la estructura burocrática promedio en Argentina, nos pone frente a un problema metodológico de consideración. Aun pese al valor central que este componente tiene -sobre todo a la luz que diferentes tipos de estructuras estatales crean diferentes tipos de capacidades para la acción (Evans 1995)-, lo cierto es que las diversas investigaciones consultadas disponen de datos desactualizados, ninguno de los cuales va más allá del 2005. Sobre todo, en lo relativo a tres índices clásicos: el índice de mérito, el de capacidad funcional y el índice de consistencia estratégica.

No obstante, haremos uso de estas investigaciones, con las reservas mencionadas, a título de indicadores sobre el estado total del aparato burocrático del Estado nacional y sobre el supuesto que no han existido variaciones estructurales en los años subsiguientes.

El índice de mérito mide el grado en el cual existen procedimientos sistematizados, técnicos, claros y transparentes en el proceso de reclutamiento, selección, promoción, compensación y desvinculación de empleados de un organismo público o agencia de gobierno. Sobre una escala de 0 a 100, los valores más bajos supondrían la existencia de criterios arbitrarios, discrecionales y con un fuerte componente político-clientelar, mientras que aquellos más cercanos al 100, se acercarían al ideal meritocrático weberiano.

Si bien en términos generales, el desempeño de la región es bajo, Argentina se ubica en un segundo grupo con valores que oscilan entre 30 y 55, junto a Colombia, México, Uruguay y Venezuela, donde fuertes componentes de prácticas clientelares conviven con mecanismos paralelos de contratación, así como se evidencian niveles altos de politización del servicio civil en el proceso de toma de decisiones de contratación, promoción y despido. Un dato coincidente con lo planteado por Andrenacci (2015).
El segundo de los índices es el relativo a la capacidad funcional de la burocracia, el cual evalúa cómo y cuánto las prácticas y patrones vigentes influyen sobre los comportamientos de los empleados, y si los mismos favorecen o no el compromiso para con la institución.

El promedio de la región en términos de capacidad funcional es de 30 (sobre 100), con picos de 61 para el caso de Brasil y un mínimo de 11 para el de Honduras (Zuvanic y Iacoviello 2005). Nuevamente, el caso argentino y venezolano no distan sustancialmente entre sí, posicionados en el rango entre 35 y 50 puntos porcentuales. Según Zuvanic y Iacoviello esta performance indicaría fuertes inequidades internas en términos salariales, a pesar de haber realizado algunos ordenamientos en la materia. Asimismo, la evaluación de desempeño se practicaría únicamente con intenciones ceremoniales y no constituye una herramienta de gestión confiable que permita distinguir entre desempeños buenos y regulares.

En suma, tal y como sostienen los autores, a partir del análisis agregado de ambos índices, existe un grupo de países calificados de "intermedios", compuesto por Venezuela, México, Colombia, Uruguay, Argentina y Costa Rica, cuyas “(...) burocracias están relativamente bien estructuradas, con cierta permanencia de funcionarios en algunos cargos, ya que se conquistó la inamovilidad para un porcentaje importante de ellos. No obstante, no se han consolidado varias de las garantías de mérito, ni las herramientas de gestión que permiten una efectiva utilización de las competencias de los sujetos, los grupos, y las instituciones" (Zuvanic y Iacoviello 2005:26)

Por último, un tema importante es el índice de consistencia estratégica, que mide la vinculación entre los procesos y prácticas de gestión de las burocracias y las prioridades estratégicas de los gobiernos. En esta línea, una vez más, Colombia, Uruguay, Argentina, Costa Rica, México y Venezuela constituyen un segundo grupo donde, si bien la conexión entre prioridades estratégicas del gobierno y la gestión de la burocracia es débil, entre 35 y 45 puntos porcentuales, tampoco se encuentra en niveles de desconexión total, como los casos inferiores a los 15 puntos porcentuales.

En este sentido, puede decirse que el sistema de servicio civil argentino presenta ciertas fugas y pérdidas entre las instancias de tomas de decisión y la ejecución misma de los programas. Un tema central al momento de analizar la forma específica que asumirá la relación entre el Estado en acción, la burocracia en términos de Oszlak (Oszlak 2006), y la sociedad. Se trata de un atributo clave que define, a nuestro entender, la necesidad de recurrir a grupos informales, por fuera de la estructura del Estado, para el cumplimiento de los objetivos.

Analizadas las cuestiones estructurales, con las reservas del caso por la disponibilidad de datos, queda un último aspecto en este componente, vinculado con las prácticas de los funcionarios en los que han sido denominados patrones de gestión pública. Los mismos pueden ser entendidos como la manifestación en superficie de una forma particular de gestionar 
políticas públicas y el resultado de la sedimentación de diversas prácticas a lo largo de los años (Andrenacci 2015:2). En un estudio exploratorio, en este sentido, edificado sobre la base de datos de observación y participación (no sistemática) en diversos procesos, Andrenacci busca desarrollar una guía de análisis con el objeto de “(...) diferenciar éxitos de fracasos en políticas públicas y calificarlos con algún parámetro de intensidad, describir sus características, trayectorias, secuencias y patrones; $y$ tratar de identificar variables a las cuales atribuir la mayor responsabilidad causal posible en situaciones de éxito y fracaso" (Andrenacci 2015:3).

Se trata de un conjunto de problemáticas ubicadas en una zona indeterminada entre factores estructurales, prácticas formales y codificadas al interior de una agencia de gobierno, y las trayectorias personales, ambiciones e intereses de los funcionarios que la componen. Un campo de cuestiones emergentes en una zona de confluencia entre problemas estructurales de capacidad estatal (aspectos institucionales, operativos, de coordinación o administrativos, medios financieros, cultura organizacional, capacidad y capacitación de los recursos humanos) y aspectos contingentes que derivan de las capacidades y atributos individuales de actores singulares o colectivos, como funcionarios, coaliciones, particos políticos, entre otros.

En este orden de ideas, puede decirse que Precios Cuidados implica un desvío del grado cero de las políticas de gestión, conforme la descripción de Andrenacci (2015:18) quien sostiene que la “(...) evolución favorable de los recursos, la decisión de enfrentar los problemas importantes largamente irresueltos, o las aproximaciones gerenciales innovadoras que han caracterizado a las gestión pública en los últimos años, parecen haber afectado áreas restringidas del Estado" (Andrenacci,2015:21), dentro de las cuales, Precios Cuidados, a nuestro entender se reservaría un lugar.

En este sentido, puede decirse que, desde el punto de vista del momento de diseño, la SCI dispuso de información para el diagnóstico, así como para ponderar entre diversas opciones, a partir de la capitalización del saber-empresarial, como fruto de las mesas formales e informales de negociación con los mismos. Incluso, la reducción de la cuestión al problema a la estructura de costos y los márgenes de rentabilidad, así como al abastecimiento y la señalización de los productos, brindó un marco simple no sólo para dar visibilidad al mismo, sino también a sus nudos críticos y factores causales: en un complejo entramado de relaciones, las agencias del Estado y sus aliados por fuera del aparato estatal, sólo debían focalizarse en la presencia/ausencia de los productos, la correspondencia/no-correspondencia de precio y la señalización efectiva en góndola. Un hecho que reduce las necesidades de mecanismos formales y efectivos de planificación.

A su vez, la articulación con redes informales brindó los mecanismos (informales) de seguimiento y evaluación necesarios, como complemento de facto de los poderes investidos en las agencias de control por la normativa preexistente al acuerdo.
Por otra parte, en cuanto a la ejecución, los recursos financieros no verificaron cambios sustanciales con etapas previas a su puesta en funcionamiento. Un punto que se complementa con una economía de autorizaciones jurídicas y permisos legales. Al respecto, se evidencia del análisis comparado de los presupuestos del Ministerio de Economía para los asientos vinculados con la "definición de políticas de comercio interior" (30), "defensa de la libre competencia" (31) y "defensa del consumidor" (35), un incremento entre 2011 y 2015 en tan sólo un 32\% más. Un aumento marginal si considera que la inflación oficial, consolidada, para el mismo período fue de $55,1 \%$ (9,5\% en 2011,10,8\% en $2012,10,9 \% 2013$ y $23,9 \%$ en 2014).

Incluso, en lo relativo al personal contratado, los dos saltos más significativos en cuanto a cantidad de empleados, se verificarían en 2012, con anterioridad a la puesta en vigencia del programa.

Por otra parte, según fuentes de la SCI, el presupuesto contempló ciertas novedades en materia de instrumentos de gestión. En este sentido, desde 2012 al 2015 se contempló como principales metas físicas e indicadores la verificación y/o aprobación de 25.000 instrumentos de medición, la realización de 30.000 operativos sobre lealtad comercial y la realización de 6 publicaciones de precios relevantes. Sin embargo, a partir de 2015 se sumaría a los anteriores la asistencia técnica a provincias, a partir de la realización de 300 talleres, 41 subsidios a entidades de consumidores, la instrucción de 495 sumarios a empresas y 1.050 casos de arbitraje entre consumidores y empresas. Indicadores no sólo más concretos, sino también cristalizaciones del rol de mediación entre consumidores y empresas que asumía el Estado, así como de monitoreo y control de las mismas.

\subsection{Acción Interinstitucional: dirección y coordinación}

Por último, en lo referente al cuarto componente, el capital de acción interinstitucional, el programa evidenciaría ciertos problemas de coordinación entre jurisdicciones. De hecho, las jurisdicciones provinciales quedarían relegadas a un rol menor, aun cuando se hayan contemplado instancias de asistencia técnica entre la Nación y las Provincias.

Sólo algunos distritos se sumaron de forma errática al control de precios, en muchos casos con listados equivocados, descargados de la página online del programa: "Muchos Intendentes y organismos de defensa del consumidor provinciales y municipales se quisieron sumar, sobre todo al principio, al ver el nivel de trascendencia mediática que tenía el programa y lo hicieron de forma poco coordinada con la Secretaría de Comercio, con listados equivocados, de otras jurisdicciones o formatos que no aplicaban a la realidad local", detalla una fuente en la coordinación de la Asociación de Supermercados Unidos. 
No obstante, según el Secretario de Comercio de una de las provincias, existían compromisos informales con la Nación para efectuar controles. En esta línea, todas las semanas -entre lunes y miércoles- se realizaban informes a la SCI sobre el cumplimiento local del programa, como parte de un compromiso más amplio (no escrito) por prorrogar las deudas provinciales con el Estado Nacional.

De esta forma, las jurisdicciones se incorporaron a la instancia de control de la ejecución, sin ningún tipo de dirección por parte de la Secretaría de Comercio, con el único requerimiento de presentar informes semanales, y superponiendo sus funciones tanto a los relevadores como a las organizaciones sociales, sin que exista legislación local que de sustento a su accionar. Incluso, en otros casos, como la definición de canastas de temporada como la "Canasta de Navidad de Precios Cuidados" o la "Canasta de Vuelta al Cole de Precios Cuidados", muchas jurisdicciones superpondrán acuerdos regionales con las cadenas locales, a aquellos establecidos por la Secretaría ("El Gobierno de San Luis...", s/f; y “El sábado lanzan...", 2014).

En palabas de un empresario del sector:"durante los primeros meses, el programa no sólo tuvo aceptación, sino que también concentró la atención de muchos medios locales en el piso de venta de nuestras sucursales. La política municipal se empezó a jugar entre nuestras góndolas y recibíamos visitas recurrentes de Intendentes y funcionarios, con periodistas, fotógrafos y sus equipos de comunicación".

A falta de coordinación interinstitucional y dirección, fue el apoyo de la opinión pública, el lugar del programa al interior de la agenda mediática y la continuidad -al momento de su ejecución- del entramado de control desarrollado por los actores no-estatales, algunos de los factores que empujaron a muchos distritos a sumarse a la iniciativa.

\section{Reciprocidad y disciplinamiento}

Recapitulando, entones, el caso argentino supuso una modalidad de intervención en la economía que, a través de los canales existentes de diálogo y negociación con el sector privado, amplió las capacidades del Estado a partir de la transferencia de recursos y la articulación con redes informales, en un pacto implícito con los grandes grupos económicos de comercialización de bienes, los cuales ganarían participación de mercado frente a los pequeños comercios tradicionales (Quiroga 2015). De hecho, el programa, lejos de restringir las condiciones de mercado y romper todo canal de conectividad con el sector privado, como en el caso venezolano, mantuvo condiciones de competitividad (en un contexto de incertidumbre económica) para los grandes jugadores, a condición de sacrificar rentabilidad y transferir recursos a su puesta en funcionamiento.
Su éxito relativo provino de una economía jurídica-administrativa sorprendente, una sensibilidad del sujeto estatal y sus agencias ante las demandas de cierta parte del empresariado del retail local en el marco de una política a la vez regulatoria y redistributiva, con alta visibilidad y bajo costo para las agencias estatales, un consecuente rédito político y un estructura burocrática mínima en articulación con empresarios, por un lado, y organizaciones sociales y políticas, por otro.

Como consecuencia, siempre desde la óptica del sujeto estatal, sus capacidades se incrementaron, como fruto de la articulación con redes informales de monitoreo, control y sanción; la intervención de forma directa en el piso de venta de las principales cadenas de comercialización; el incremento en el flujo de intercambio de información del sector privado a las agencias de gobierno involucradas; y la transferencia indirecta de recursos del sector privado a la operación del programa, como garantía de su sustentabilidad de mediano plazo.

En este marco, y como fruto de su éxito relativo, se incrementó además la legitimidad del sujeto estatal en tanto regulador e interventor sobre las fuerzas de mercado (garantizada incluso por la conformidad del empresariado); se reforzaron los vínculos con sujetos no-estatales; y se desarrollaron nuevos arreglos institucionales para compensar los déficits históricos del aparato estatal.

Este punto contrasta sustancialmente, como se evidencia en el siguiente cuadro, con el programa venezolano de control de precios, el cual enfrentó a las agencias estatales al empresariado a partir de la movilización de todo el aparato represivo del Estado, en el marco de una "Guerra Económica". Precios Cuidados, en cambio, puso en acto instancias de "disciplinamiento" del empresario, en los términos de Amsden (1992): una componente central de la reciprocidad en la relación entre Estado y empresarios, sobre la base de normas claras de desempeño fijadas por los funcionarios; acceso confiable y preciso a la información para poder evaluar, que lo funcionarios tengan capacidad de imponer sanciones; y mecanismos de control social.

Incluso, la particularidad de la forma Precios Cuidados, sobre todo en lo relativo a la articulación con actores privados, es lo que posibilitó escapar a los efectos propios de las "trampas" implicadas en las políticas de control de precios y subsidios, como fue el caso de las tarifas de servicios públicos en Argentina. En este sentido, la propuesta de "policy trap" que formulan Mascarenhas y Post (2014), suponía que las políticas de control de precios a través de subsidios eran puestas en marcha a un costo relativamente bajo, pero que con el tiempo reducen el margen de acción de los gobierno por los desincentivos y costos de su desarticulación. 
Figura 2: Comparación de las modalidades de articulación entre Estado y Sociedad de los programas en Venezuela y Argentina

\begin{tabular}{|c|c|c|}
\hline & Modalidad Venezolana & Modalidad Argentina \\
\hline Dispositivos de denuncia & $\begin{array}{l}\text { - Línea telefónica } \\
\text { - Portal web }\end{array}$ & $\begin{array}{l}\text { - Línea telefónica } \\
\text { - Portal web } \\
\text { - Aplicación Móvil }\end{array}$ \\
\hline Sanciones & $\begin{array}{l}\text { - Multas } \\
\text { - Privación de libertad } \\
\text { - Decomisos } \\
\text { - Ocupación temporal de empresas }\end{array}$ & $\begin{array}{l}\text { - Multas } \\
\text { - Actas }\end{array}$ \\
\hline Redes formales & $\begin{array}{l}\text { - Fuerzas Armadas } \\
\text { - Fiscales de Estado } \\
\text { - Reunión con empresarios "Comisión } \\
\text { de la Paz del Área Económica" }\end{array}$ & - 41 inspectores \\
\hline Redes Informales & $\begin{array}{l}\text { "Fiscales parroquiales" } \\
\text { "Brigadas juveniles para la defensa } \\
\text { popular de la economía" }\end{array}$ & - Organizaciones sociales y militantes \\
\hline Sistemas de formación & $\begin{array}{l}\text { - Publicidad oficial } \\
\text { - Portal web } \\
\text { - Talleres }\end{array}$ & $\begin{array}{l}\text { - Publicidad Oficial } \\
\text { - Portal web } \\
\text { - Intervención de piso de venta }\end{array}$ \\
\hline \multirow[t]{2}{*}{ Alianza entre sectores } & Estado + Organizaciones Sociales & Estado + Empresarios + Organizaciones Sociales \\
\hline & ENFRENTAMIENTO & DISCIPLINAMIENTO \\
\hline
\end{tabular}

Fuente: elaboración propia

En el caso referido por los autores, entre el 2003 y el 2008, se habría registrado una estrategia institucional de creciente involucramiento estatal, a partir de un programa sustentado en transferencias crecientes de recursos al sector privado, con el subsidio como el principal instrumento, y escasos sistemas de rendición de cuentas y control de las empresas. La segunda etapa, en cambio, implicaba una estrategia institucional de búsqueda por disminuir el involucramiento estatal y la presión fiscal, a partir de la revisión del subsidio como instrumento único y central, para la instrumentalización de sistemas online de información, monitoreo y de control de los mismos, así como segmentación de los usuarios para la revisión de los beneficiarios.

Sin embargo, para el caso de Precios Cuidados, aun manteniendo las mismas condiciones contextuales y objetivos de corto plazo (a saber: contener el "humor social" y enfrentar escenarios de volatilidad electoral), no existieron tensiones de corto y mediano plazo en relación a los costos porque el financiamiento de la brecha de precio entre el "precio cuidado" y aquel librado a las fuerzas de mercado, fue saldado por las empresas productoras y comercializadoras. Precisamente, por el carácter redistributivo del programa que analizábamos con anterioridad.

De esta forma, a diferencia de los subsidios al consumo de servicios públicos, la sustentabilidad de mediano y largo plazo del consumo minorista no se vió afectada: la política pública de control no se vuelve cara en términos de recursos fiscales, ni requiere necesariamente de una mayor inversión. Sus resultados, tampoco se vieron limitados por la escasa visibilidad de la misma y el costo de "sinceramiento", como se ha dado en llamar mediáticamente al reconocimiento de los precios de mercado.

Por el contrario, el estatuto de Precios Cuidados, al interior del conjunto de políticas de control de precios y subsidio al consumo, radica en que lejos de tener poca visibilidad social, alto costo y difícil rédito político, posibilitó una alta visibilidad a partir de la construcción misma de una marca, un alto rédito político con índices de aprobación del orden del 63\% a noviembre de 2014, como fruto de la distribución amplia de beneficios y la concentración de los costos en pocas empresas, así como la distribución universal de sus beneficios. En los términos de consultores privados: "Si hay algo para reconocer es que el Gobierno es un gran creador de marcas. Esto se puede ver no sólo con Precios Cuidados, sino también en propuestas como Ahora 12 o Procrear, que, más allá de las críticas, gozan de una alta adhesión en la sociedad" (Sainz, 2015).

\section{Conclusiones: el síntoma de la estatalidad}

Precios Cuidados ha sido la iniciativa política de un Estado en el marco de una coyuntura caracterizada por inflación creciente y su impacto en la opinión pública, con un consecuente deterioro de la legitimidad institucional del Estado y la legitimidad política de la coalición gobernante.

Las particularidades de su forma, por oposición al caso venezolano, posibilitó el mantenimiento de canales de diálogo con el sector privado y la institucionalización (incipiente) de instancias de comunicación entre el Estado, y sus agencias, y el empresariado, sin necesidad de recurrir a mecanismos de coerción directa (garantizadas, fundamentalmente, por la necesidad de negociar de forma periódica los términos y alcances del programa). Particularidades que constituyeron un factor decisivo en el éxito (relativo) del programa y que tuvo -como condición de posibilidad- un componente clave de reciprocidad para con el empresariado: la oferta a los sectores decisivos de la producción y la distribución de un esquema de beneficios claros como condición para ganar participación de mercado a cambio de renunciar a márgenes de rentabilidad.

Sin embargo, más allá del caso particular, lo cierto es que Precios Cuidados es la manifestación en superficie de un conjunto de problemáticas subyacentes comunes a todas las políticas de regulación del mercado de precios de bienes de consumo masivo, por tratarse de un tema de alta sensibilidad pública y que hacen de este campo de políticas públicas un lugar privilegiado para el estudio de las relaciones del Estado con su otredad.

Después de todo, el problema de las políticas de control de precios nos enfrenta al diseño de instrumentos (políticos) no sólo para la administración pública de los flujos de relaciones económicas, sino que también constituyen un condicionamiento de base 
para la accesibilidad/no-accesibilidad a una serie prácticas que exceden lo económico: el precio de los alimentos de consumo, después de todo, nos sitúa frente a problemas políticos, como la constitución de alianzas de clase; a dimensiones sociológicas, como las cuestiones relativas a los sistemas de producción y distribución de los alimentos; y dimensiones antropológicas, en vistas a que no se trata sólo de la posibilidad de acceder a bienes de consumo para reproducir la fuerza de trabajo y saciar el hambre, sino que involucra sistemas de valores, hábitos y costumbres que hacen a la identidad de los pueblos. Nos pone frente a gustos relativos a la nacionalidad, la identidad, la edad, la religión y el sexo, así como la división sexual del trabajo al interior de las unidades domésticas. En suma, puede decirse que, con anterioridad al pasaje de lo crudo a lo cocido, y los mitos que lo rodean, el precio de los productos es la medida de acceso/no-acceso a este conjunto de relaciones sociales.

La discusión sobre el valor de los bienes de consumo se inserta al interior de este denso entramado de relaciones y abordajes, que exceden al hecho económico y nos pone frente a la discusión de los límites de la capacidad absoluta del Estado, así como de sus agencias para intervenir el tejido social de forma coordinada y consistente. Cada vez más su realización, la de sus políticas y fines, dependen de la articulación con actores no estatales, de prácticas informales y recursos ilegales.

Llegamos entonces, a la identificación con el síntoma: en el barro de la historia de las capacidades del Estado, es sus pliegues, en sus falencias, en su funcionamiento híbrido, al límite de la legalidad misma que sanciona, no encontramos un desvío o una anomalía, sino más bien, la verdad de su funcionamiento. O más aún: la condición de su eficacia.
${ }^{1}$ Conforme a los datos provistos por la Ficha Técnica del estudio en cuestión, se trata de una investigación cuantitativa, con un cuestionario semi estructurado compuesto por preguntas abiertas, con una duración aproximada de 25 minutos. La muestra total fue de 998 casos, tomada en todo el país entre hombres y mujeres, entre 16 y 75 años de todos los NSE, con un método de muestreo por cuotas de sexo, edad, nivel socio económico y región. Por último, el error muestral se calculó en $+/-3.09 \%$ y un nivel de confianza del $95 \%$.

${ }^{2}$ Resolución 2/2014. Boletín Oficial, 12 de enero 2014.

3 La referencia a "items comparables" supone que como la canasta de Precios Cuidados pasó de 194 productos enero a administrar 506 en 2015, la base comparable solo se hace sobre aquellos productos presentes en tos enero a admin
ambos períodos.

${ }^{4}$ El "escrache" se trata de un método de protesta de acción directa por el cual se busca visibilizar a los responsables (a partir de manifestaciones frente a sus viviendas o publicando sus fotos, entre otras opciones) de un presunto delito o injusticia a los ojos de determinadas organizaciones sociales o activistas. 


\section{Referencias}

Adams, Richard (2000) Self-Targeted Subsidies:The distributional Impact of the Egyptian Food Subsidy System. Washington, DC: World Bank Policy Research Working Paper 2322.

Agenor, Pierre-Richard y Asilis, Carlos (1997). "Price controls and electoral cycles". European Journal of Political Economy, vol. 13(1): 131-142.

Alderman, Harold (2002). Subsidies as a social safety net: Effectiveness and challenges. Washington, DC: World Bank.

Alonso, Guillermo (2000) Política y Seguridad Social en la Argentina de los 90. Buenos Aires: Miño y Dávila Editores.

Amengual, Matthew (2011). "Cambios en la capacidad del Estado para enfrentar las violaciones de las normas laborales. Los talleres de confección de prendas de vestir en Buenos Aires”. Desarrollo Económico 51:202-203.

Amsden, Alice H. (1992). Asia's Next Giant: South Korea and Late Industrialization. New York: Oxford University Press.

Andrenacci, Luciano (2015). "Una aproximación a la "mala gestión” en políticas públicas. Los déficits en la gestión de la información; el intuicionismo y la sobrepolitización en el Estado argentino". En VIII Congreso de la Asociación Latinoamericana de Ciencia Política (ALACIP). Lima. Inédito.

Bertranou, Julián (2013). "Capacidad estatal. Aportes al debate conceptual”. En $7^{\circ}$ Congreso Argentino de Administración Pública: Mendoza. Inédito.

Bienen, Henry. S. y Gersovitz, Mark (1986). "Consumer subsidy cuts, violence, and political stability”. Comparative Politics, 19 (1), 25-44.

Birkland, Thomas A. (2005) An Introduction to the Policy Process. Theories, concepts and models of public policy making. Armonk: M.E. Sharpe.

Cheung, Steven (1974) "A Theory of Price Control”, Journal of Law and Economics,Vol. 17 (1) $53-71$.

Colander, David (1984). "Galbraith and the Theory of Price Control". Journal of Post Keynesian Economics vol. 7 (1) 30-42.
Cuyo Noticias (2014) "El gobierno de San Luis acordó una canasta escolar". Recuperado de http:// http://cuyonoticias.com/2014/21557

]

Dewey, Matías (2012) "The making of Hybrid Stateness: sources of Police Performan ce in the Conurbano". Revista de Ciencia Politica, 32 (3), 599-611, Santiago de Chile.

Diario Jornada (2014) "El sábado lanzan en Chubut la Canasta Navideña" (3 de diciembre). Recuperado de http://www.diariojornada.com.ar/113070/politica/El_6 lanzan_canasta_navidea_en_Chubut_con_20_productos

Diario UNO (2014) "Confirmaron multas a supermercados por violar los 'precios cuidados"' 9 de diciembre. Recuperado de http://www.diariouno.com.ar/pais/confirmaron-multas-supermercados-violar-los-precios-cuidados-20141209-n154843.htm

Dunn, Stephen y Pressman, Steven (2005) “The Economic Contributions of John Kenneth Galbraith”. Review of Political Economy, 17(2), 161-209.

Echebarria, Koldo y Cortazar, Juan Caros (2006). "Las reformas de la administración y el empleo público en América Latina". En El estado de las reformas del Estado en América Latina. Lora, Eduardo (ed). Washington: Banco Interamericano de Desarrollo, pp. 139-173.

Echebarria, Koldo (2006). "Caracterización empírica de las burocracias latinoamericanas: configuraciones y roles en el proceso de elaboración de políticas públicas". Revista del CLAD Reforma y Democracia, No. 34:1-11.

Evans, Peter (1995). Embedded Autonomy: States and Industrial Transformation. Princeton: Princeton University Press.

Evans, Peter y Rueschemeyer, Dietrich (1985) "The Sate and Economic Transformation: Toward an Analysis of the Conditions Underlying Effective Intervention”. En Peter Evans, Dieter Rueschemeyer y Theda Skocpol (eds.), Bringing the State Back In New York: Cambridge University Press, 3-43.

FIEL (1990) Control de precios e inflación. La experiencia argentina reciente. Buenos Aires: FIEL.

Franco, Liliana (2014) "Precios Cuidados: Kicillof anunció multas por \$31 millones a supermercados". Ámbito Financiero, 9 de mayo. Recuperado de http://www.ambito. com/noticia.asp?id=740152 
Friedman, Milton (1977) "From Galbraith to Economic Freedom", Occasional Paper 49, London: Institute of Economic Affairs.

Friedman, Milton \& Friedman, Rose. D. (1990) Free to choose: a personal statement San Diego: Harcourt Brace Jovanovich.

Galbraith, John Kenneth (1941)."The Selection and Timing of Price Controls", The Review of Economic Statistics, 23(2), 82-85.

Galbraith, John Kenneth (1946) "Reflections on Price Control", The Quarterly Journal of Economics 60 (4) 475-489.

Galbraith, John Kenneth (1951) "The Strategy of Direct Control in Economic Mobilization", The Review of Economics and Statistics, 33(1), 12-17.

Galbraith, John Kenneth (2001) The Essential Galbraith. Boston : Houghton Mifflin.

Mascarenhas, Tomás Bril y Alison E. Post (2014), "Policy traps y subsidios al consumo. La política de tarifas de servicios públicos en Argentina, 2002-2014”. Desarrollo Económico 54 (213) 171-202.

Mora y Araujo, Manuel (2011) La Argentina bipolar. Las variantes de la opinión pública (1983 - 2011), Buenos Aires, Sudamericana.

North, Douglass (1990). Institutions. Institutional Change and Economic Performance, Cambridge: Cambridge University Press.

O’Donnell, Guillermo (1977) “Estado y alianzas en la Argentina, 1956-1976”. Desarrollo Económico 16 (64): 523-5

Oszlak, Oscar (2006) "Burocracia estatal: política y políticas públicas". Revista POSTData, $\mathrm{N}^{\circ} 11$, Abril 2006. www.oscaroszlak.org.an

Pierson, Paul (1993) "When Effects Become Cause: Policy Feedback and Political Change". World Politics, Vol 45 (4) 595-628.

Quiroga, Juan Pablo (2015) Control(es) de Precio y capacidades estatales. Disciplinamiento, transferencia y redes informales de control, monitoreo y sanción. El caso de Precios Cuidados en Argentina y su aceptación por parte del empresariado del retail local. (Tesis no publicada). Georgetown University, Washington D.C.
Richardson, Neal O. (2009) "Export-oriented populism: commodities and coalitions in Argentina”. Studies in International Comparative Development 44 (3) 228-255.

Rockoff, Hugh (1984) Drastic Measures: A History of Wage and Price Controls in the United States. New York: Cambridge University Press.

Sainz, Alfredo (2015) "Precios cuidados: un éxito de marketing, un fracaso para frenar la inflación". La Nación (20 de mayo). Recuperado de http://www.lanacion.com ar/1794252-un-exito-de-marketing-un-fracaso-para-frenar-la-inflacion

Saylor, Ryan (2012) "Sources of State Capacity in Latin America: Commodity Booms and State Building Motives in Chile". Theory and Society 41(3) 301-324.

Schuettinger, Robert y Butler, Eamonn (1978) Forty Centuries Of Wage And Price Controls: How Not to Fight Inflation, Washington, D.C.: The Heritage Foundation.

Taussig, Frank (1919) "Price Fixing as seen by a Price-Fixer", Quarterly Journal of Economics, 33(2) 205-241.

Vogel, David (1987) "Political Science and the Study of Corporate Power: A Dissen from the New Conventional Wisdom”. British Journal of Political Science 17 (4):385-408.

Weber, Max (1947) Economía y Sociedad. México: Fondo de Cultura Económica.

Zuvanic, Laura y Iacoviello, Mercedes (2005) "La burocracia en América Latina", ICAP-Revista Centroamericana de Administración Pública, No 58-59, págs. 9-41.

\section{Cómo citar este artículo:}

Quiroga, Juan Pablo (2017) "El síntoma de la estatalidad: controles de precios y las capacidades estatales. El caso de Precios Cuidados en Argentina". Revista Perspectivas de Políticas Públicas vol. 7 № 13 